\title{
Tipos de estudio en el enfoque de investigación cuantitativa
}

Mtra. María Cristina Müggenburg Rodríguez V.* y Mtra. Iñiga Pérez Cabrera**.

* Secretaria de Asuntos del Personal Académico de la ENEO-UNAM

** Profesora de Carrera Asociado "C" de Tiempo Completo de la ENEO-UNAM

La preparación de clases para impartir la materia de Metodología de Investigación del programa de Licenciatura de la Escuela Nacional de Enfermería y Obstetricia de la Universidad Nacional Autónoma de México implica la revisión de diversos autores, en este proceso nos encontramos con criterios diferentes para la presentación de los temas, en el presente caso nos abocamos a los tipos de estudio, para el desarrollo de este contenido identificamos una amplia gama de presentación, por lo que nos decidimos a revisar y organizar el material bajo diversos criterios como profundidad de la búsqueda, fuente de datos, intervención y propósito del investigador, disponibilidad de tiempo y recursos, etc. Sin embargo, es importante mencionar que estas clasificaciones no son absolutas y que un estudio puede ubicarse en más de un grupo si el criterio establecido lo incluye.

PALABRAS CLAVE: Tipos de estudio, Investigación cuantitativa..

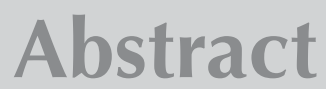

The preparation of our classes implies the revision of diverse authors, in this process we were with criteria different for the presentation from the subjects, in the present case we were led the types of study, for the development of this content we identified an ample range of presentation, reason why we were decided to review and to organize the material under diverse criteria like depth of the search, source of data, intervention and intention of the investigator, availability of time and resources, etc. Nevertheless, is important to mention that these classifications are not absolute and that a study can be located in more of a group if the established criterion includes it.

KEY WORDS: Types of study, Quantitative investigation

El ser humano con su capacidad de razonar, de elegir y trascender se plantea dudas y busca respuestas con el fin de conocer, comprender y enfrentar un mundo con el deseo de transformar la realidad.

La toma de conciencia de un problema susceptible de ser estudiado conduce al desarrollo del proceso de investigación, mismo que implica una secuencia evolutiva que persigue un fin que puede ser la obtención de un conocimiento, la explicación de un hecho, la predicción del comportamiento de un fenómeno o bien la solución de un problema.

En este contexto como profesores de la materia de Metodología de Investigación del programa de la Licenciatura en Enfermería y Obstetricia con enfoque cuantitativo nos hemos encontrado con diversas posturas de los autores que apoyan nuestra docencia, sobre todo en algunos temas, motivo por el cual y como resultado de las revisiones bibliográficas y de la necesidad de ofrecer orientaciones más claras a nuestros alumnos, hemos tomado la decisión de abordar en esta presentación los diferentes tipos de estudio de investigación, mismos que responden al problema, a los objetivos, a la fuente de datos, a la experiencia del investigador, a las revisiones bibliográficas y al tiempo y recursos disponibles para llevar a cabo el estudio.

Tomando en cuenta diversas clasificaciones sobre tipos de estudio de investigación, se organizan bajo diferentes criterios, sin dejar de reconocer que estas clasificaciones no son absolutas y que un estudio puede ubicarse en más de un grupo si el criterio establecido lo incluye. 
1) De acuerdo con el nivel de profundidad de la búsqueda planeada del conocimiento que se pretende obtener:

- Descriptivo

- Explicativo ( o analítico)

- Predictivo (experimental). ${ }^{1}$

1.1.) En el ámbito de los Estudios descriptivos se pueden mencionar: a) descriptivo típico (describe las características de una sola muestra); b) descriptivo comparativo (describe las diferencias de variables en dos o más grupos); c) estudio de caso (descripción intensa de una unidad de estudio). ${ }^{2}$

1.2) Estudios explicativos o analíticos: explican, contestan el porqué o la causa de presentación de determinado fenómeno o comportamiento, se trata de explicar la relación o asociación entre variables. ${ }^{3}$ y 4

Pineda reclasifica estos estudios en cohortes y controles, pero los autores de esta presentación establecen otro grupo independiente que a su vez puede estar subsumido en los explicativos.

Burns y Grove, aportan para este grupo al diseño correlacional descriptivo(describe las variables y examina las relaciones entre ellas). ${ }^{5}$

1.3) Estudios predictivos: predicen, estudian comportamientos controlando intervenciones y analizando resultados en diferentes condiciones con el fin de establecer efectos predecibles que ofrecen elementos para el establecimiento de normas y controles.

2) De acuerdo con la intervención del investigador sobre el fenómeno estudiado.

- Observacional

- Experimental

2.1) Estudios observacionales: se sustenta en el uso de técnicas que permiten al investigador adquirir información por medio de la observación directa y el registro de fenómenos, pero sin ejercer ninguna intervención (dejando libres a los observados).
2.2) Estudios experimentales: se caracterizan por la intervención intencionada y programada en la que se manipulan una o más variables (independientescausales) con el fin de analizar las consecuencias que esta manipulación ejerce sobre otra u otras variables (dependientes-efectos).

En el ámbito de salud se les conoce también como Ensayo clínico o como Ensayo comunitario. ${ }^{6}$

3.) De acuerdo con el momento en que ocurre el fenómeno y su registro.

- Retrospectivo

- Prospectivo

3.1) Estudios retrospectivos o retrolectivos: son aquellos en los cuales se indaga sobre hechos ocurridos en el pasado.

3.2) Estudios prospectivos o prolectivos: son aquellos en los cuales la información se va registrando en la medida que va ocurriendo el fenómeno ó los hechos programados para observar. $^{7}$

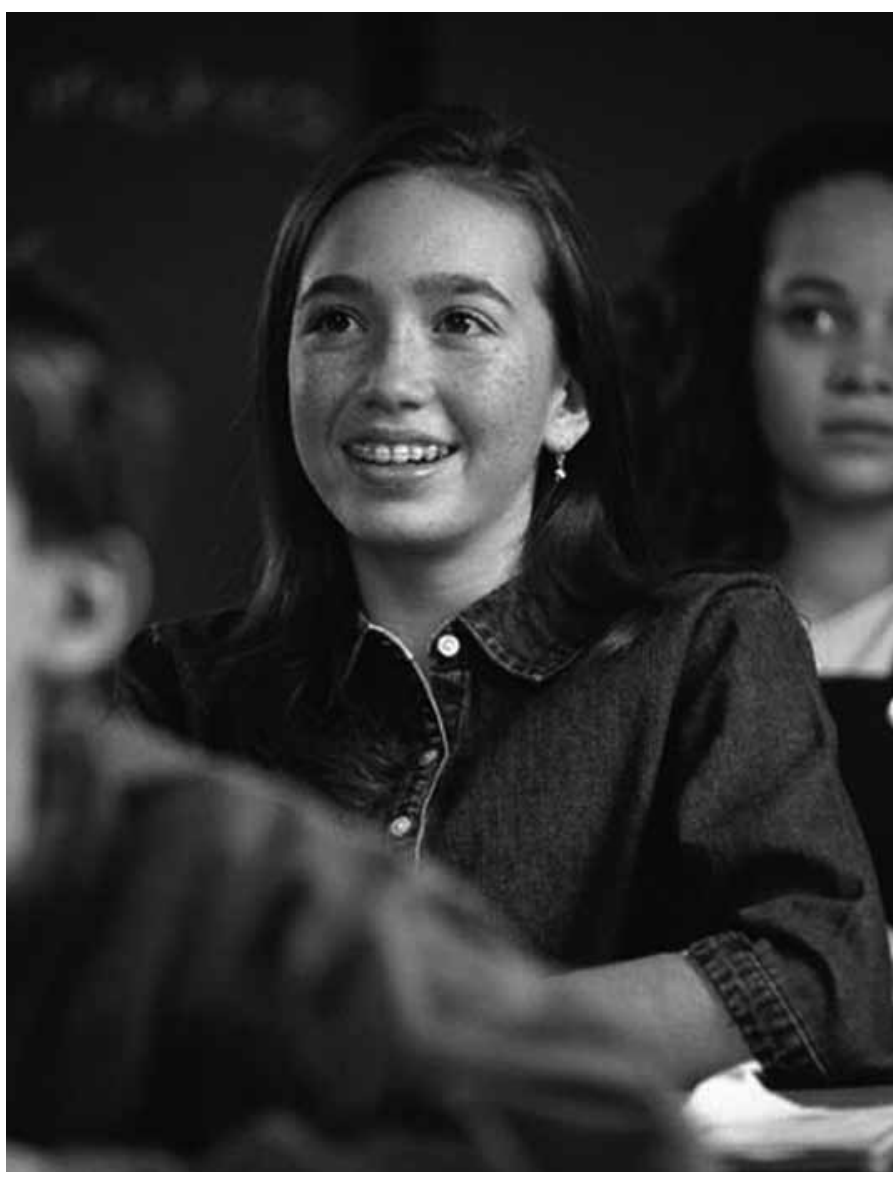


4) De acuerdo con el número de ocasiones en que se colectan los datos sobre el fenómeno estudiado.

- Transversal

- Longitudinal

4.1) Estudios transversales: son aquellos en los que se recolectan datos en un sólo momento, en un tiempo único. Su propósito se centra en describir variables y analizar su comportamiento en un momento dado. (es como tomar una fotografía de algo que sucede).

También se les conoce como estudios de corte transversal

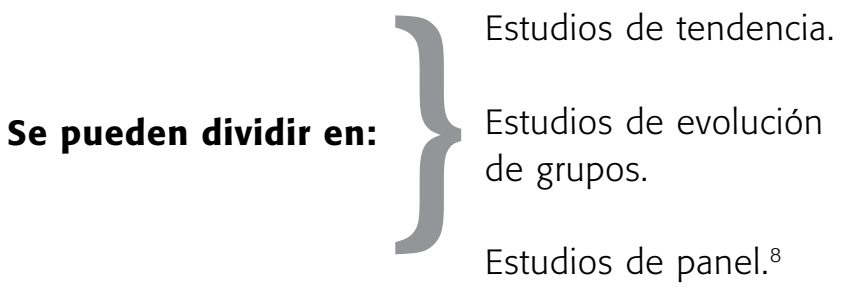

4.2) Estudios longitudinales: son aquellos en los que se recolectan los datos a través del tiempo, en períodos especificados, con el fin de hacer inferencias respecto al cambio, sus determinantes y sus consecuencias. (es como tomar una película de algo que sucede).

5) De acuerdo con el sentido de la explicación del fenómeno.

- Casos y controles (o retrospectivo)

- Cohorte (o prospectivo)

5.1) Estudios de casos y controles: estos estudios requieren del seguimiento de dos grupos: casos (con el problema o enfermedad) y controles (sin el problema o enfermedad). En este caso se toma como punto de referencia la presencia de un efecto (problema o enfermedad) y se estudia el pasado para investigar la supuesta causa:
Aquellos que tienen el efecto (problema o enfermedad) pertenecen al grupo de CASOS:

Por otra parte, se busca un grupo similar pero que no presente el efecto (problema o enfermedad), pertenecen al grupo de CONTROLES.

Ambos grupos se comparan con el fin de identificar la causa que generó el efecto.

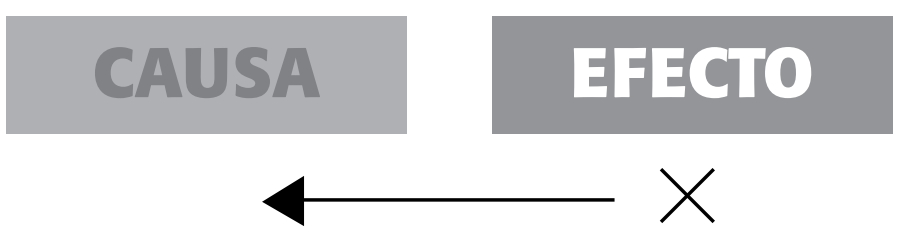

5.2) Estudios de cohorte: estos estudios examinan a una población sujeta a un riesgo a través del tiempo con el fin de identificar si determinada causa genera un efecto.

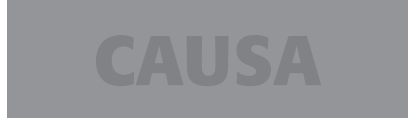

EFFECIO

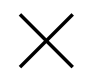

Se puede seguir una sola cohorte o grupo.

Se pueden seguir dos cohortes o grupos.

- Expuesto a la causa (casos)

- No expuesto a la causa (controles).

6) De acuerdo con la fuente de acopio de los datos.

- Documental

- De campo

6.1) Estudios documentales: son aquellos que se apoyan en registros y documentos, de los cuales se obtiene la información.

6.2) Estudios de campo: son aquellos que se llevan a cabo en el lugar de ocurrencia del fenómeno. 
7) De acuerdo con el fin último que persigue la investigación.

- Básica

- Aplicada

7.1) Investigación básica: se orienta a la acumulación de información o la formulación de una teoría. Este tipo de investigación no está encaminado a resolver problemas inmediatos, sino a la ampliación de la base de conocimientos de una disciplina por el conocimiento y la comprensión en sí.

7.2) Investigación aplicada: se concentra en la solución de un problema inmediato, ofrece elementos para aplicaciones tecnológicas o para toma de decisiones.

Ambos tipos de investigación están muy relacionadas entre sí.

\section{CONCLUSIONES}

Los grupos establecidos para clasificar los estudios de investigación de acuerdo con diferentes criterios pueden ser cuestionables, unos están más dirigidos al diseño en sí y otros a la fuente o aplicación, pero de acuerdo con el propósito didáctico de este artículo se pretende que, cuando el alumno revise a diferentes autores pueda establecer las diferencias y semejanzas entre las clasificaciones que cada uno de ellos presenta sin que esto le genere confusión.

\section{REFERENCIAS BIBLIOGRÁFICAS}

1 Polit, D y Hungler B. Investigación Científica en Ciencias de la Salud; Ed.Mc Graw-Hill Interamericana, México, 2000. p.7-18.

2 Burns, N y Grove, S.K. Investigación en Enfermería; Ed. ElsevierSaunder, $3^{a}$.ed. Madrid, 2004. p.218-221

3 Polit y Hungler Op.Cit. p. 18

4 Pineda, E.B. Alvarado y Canales. Metodología de la Investigación; Publicación de la OPS Ed OPS, $2^{\mathrm{a}}$.ed. 1994. p.84

5 Burns, N y Grove S. Op.Cit. p.7

6 Lilienfeld, A y Lilienfeld D. Fundamentos de Epidemiología; Ed. Fondo Educativo Interamericano, 1983. p.230 y 251.

7. Pineda EB Op.Cit. p.81

8 Hernández Sampieri y cols. Metodología de la Investigación; Ed. Mc Graw Hill Interamericana, 3ªed. México, 2003 p. 278284.

\section{DIRECCIÓN PARA CORRESPONDENCIA}

Cristina Müggenburg: cmuggenb@yahoo.com.mx

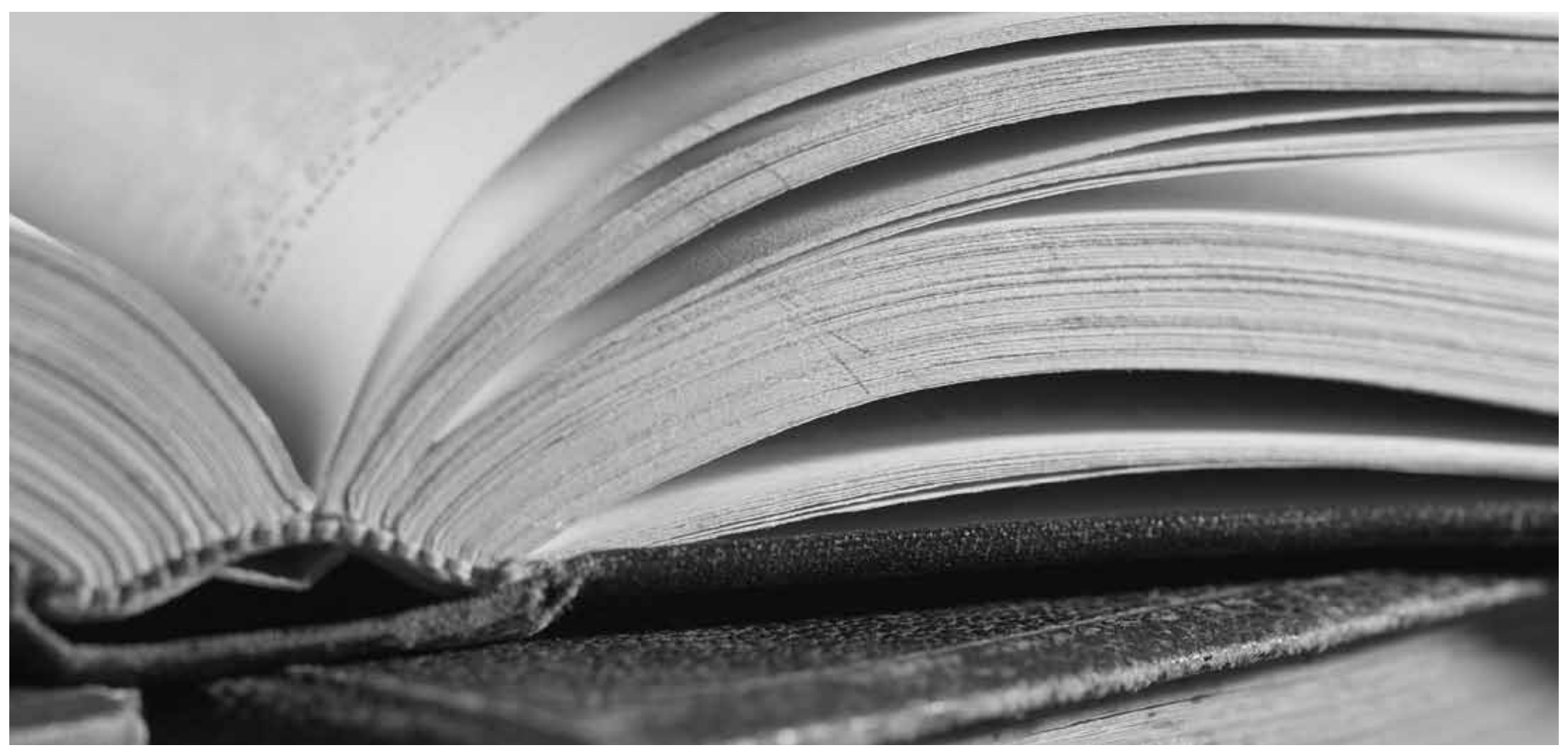

